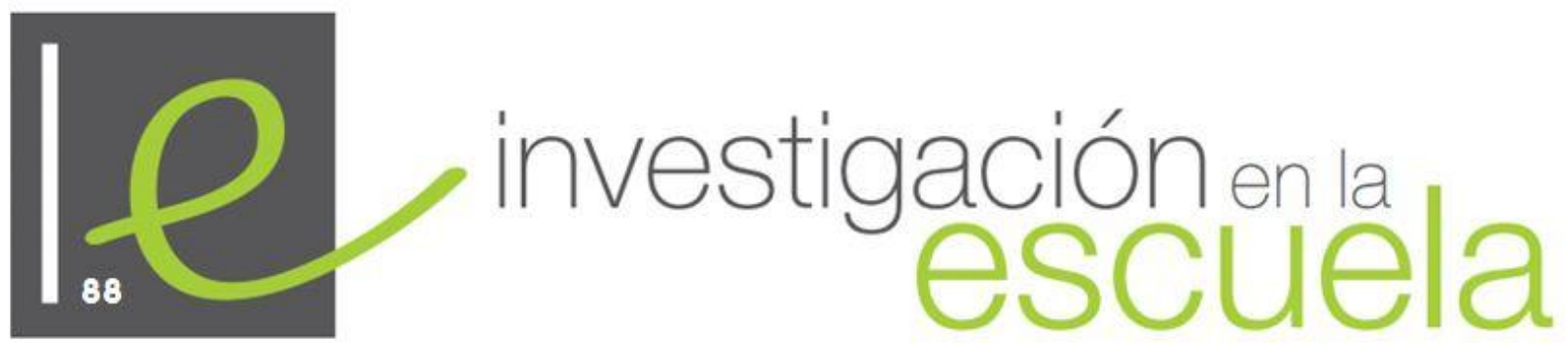

Revista académica evaluada por pares y de acceso abierto

\title{
Aprender a detectar las ideas del alumnado de Primaria sobre los contenidos escolares de ciencias
}

\author{
Emilio Solís Ramírez, Rafael Porlán Ariza, Rosa Martín del Pozo \\ y Joao Siqueira Harres \\ Universidad de Sevilla \\ España
}

Citación: Solís, E., Porlán, R., Martín del Pozo, R. \& Harres, J. (2016). Aprender a detectar las ideas del alumnado de Primaria sobre los contenidos escolares de ciencias. Investigación en la escuela, 88, 4662. Recuperado de: www.investigacionenlaescuela.es/articulos/R88/R88-3

Resumen: entre las muchas competencias que deben desarrollar los futuros maestros está el que sean capaces de detectar las ideas de los alumnos para poder utilizarlas didácticamente. El propósito de este estudio es describir y analizar la progresión de los futuros maestros al diseñar un instrumento para detectar las ideas de los alumnos de Primaria sobre contenidos de ciencias, cuando participan en cursos de formación inicial con una estrategia de orientación constructivista. Los resultados del análisis de las producciones escritas de los participantes sugieren que, al finalizar el proceso formativo, los futuros profesores son capaces de elaborar instrumentos mucho más idóneos para detectar las ideas de los alumnos y se alejan de un formato similar al examen memorístico. Finalmente, se discuten las posibles implicaciones para la formación inicial de maestros Palabras clave: "ideas de los alumnos"; "concepciones de los maestros"; "formación inicial de maestros"; "contenidos escolares"; "educación Primaria".

\section{Learning to detect students' ideas about school-level content on science}




\begin{abstract}
: among the many competences that must be developed future teachers is that they can detect the students' ideas to use them in the classroom didactically. The purpose of this study is to describe and analyze the progression of future teachers to design an instrument to identify students' ideas about Primary contents of science, when they participate in initial training with a constructivist orientation strategy. The results of the analysis of the written productions of the participants suggest that the end of the training process, student teachers are able to develop more suitable instruments to detect students' ideas and away from a format similar to rote exam. Finally, we discuss possible implications for initial teacher education.
\end{abstract}

Key words: "students' ideas, Teachers' conceptions"; "initial teacher education"; "school-level content"; "primary Education".

Apprenez à identifier les idées des élèves sur le contenu scolaires sur la science

Rèsumé: Texto de resumo Parmi les nombreuses compétences que les futurs enseignants doivent développer qui est capable de détecter les idées des élèves à les utiliser de façon didactique. Le but de cette étude est de décrire et d'analyser la progression des futurs enseignants à concevoir un instrument pour détecter les idées des élèves du primaire sur le contenu de la science, en participant à la formation initiale avec une stratégie d'orientation constructiviste. Les résultats de l'analyse des productions écrites des participants ont suggéré que les futurs enseignants sont à la fin du processus de formation, en mesure de développer des outils plus appropriés pour détecter les idées des élèves et à l'extérieur à partir d'un format similaire à l'examen par cœur. Enfin, les implications potentielles pour la formation initiale des enseignants sont discutés.

Mosts clé: "idées des élèves"; "conceptions des enseignantss"; "formation initiale des enseignants,"; "contenu scolaire"; "enseignement primaire".

\title{
Marco teórico
}

Las ideas de los alumnos sobre los contenidos escolares de ciencias tienen un gran interés en la formación inicial de maestros. Muchos autores destacan que cuando los futuros maestros se enfrentan a la detección, análisis y utilización didáctica de las ideas de los alumnos de Primaria, suele progresar su conocimiento tanto del contenido como de su enseñanza y aprendizaje, y son capaces de cuestionar el modelo transmisivo de enseñanza (Joram y Gabriele, 1998; Jones, Carter y Rua, 1999; Watters y Ginns, 2000; Morrison y Lederman, 2003).

Una parte de estas investigaciones sobre el conocimiento didáctico del profesorado se centra en el estudio de la progresión de las concepciones de los futuros profesores. En esta línea, hemos presentado los resultados del estudio de tales progresiones sobre la metodología de enseñanza (Rivero et al., 2010; Solís et al., 2012), los contenidos escolares (Martín del Pozo, Porlán y Rivero, 2011), y la naturaleza, cambio y utilización didáctica de las ideas de los alumnos (Martín del Pozo, Rivero y Azcárate, 2014).

En este artículo, nuestra aportación se centra en la descripción y análisis de la progresión de una muestra de futuros maestros al elaborar un instrumento para detectar las ideas de los alumnos cuando participan en cursos de formación de orientación constructivista.

\section{Las ideas del alumnado y la visión del profesorado}

Como señalan Treagust y Duit (2008), la investigación didáctica durante las últimas tres décadas ha demostrado que los alumnos van a clase de ciencias con ideas acerca de los fenómenos físico-naturales, que no están en consonancia con los puntos de vista que se quieren enseñar. En 
otras palabras, hay consenso en admitir que no puede considerarse a los alumnos como un saco vacío al que llenar de contenidos. Por lo tanto, los profesores tienen que darse cuenta de que sus alumnos ya tienen conocimientos propios sobre los contenidos a enseñar, que suelen ser alternativas al conocimiento que se quiere enseñar y que, en ese caso, suponen verdaderos obstáculos para la comprensión conceptual.

No obstante, existen diferentes planteamientos sobre las ideas de los alumnos relacionados con los modelos didácticos. Tal y como sugiere Giordan (1989), en un modelo didáctico de corte tradicional, las ideas de los alumnos no tienen valor epistemológico, no se considera que los alumnos tengan ideas propias, espontáneas, sino que se conciben como el recuerdo de los contenidos ya explicados en el aula. De ahí que para que esos conocimientos se amplíen, los alumnos deben incorporar nuevas informaciones. Una enseñanza transmisiva es la forma más habitual de hacerlo, y el examen memorístico un instrumento frecuente para comprobar la incorporación de tales informaciones. Esta suele ser la visión inicial de muchos maestros en formación y en activo. En el otro extremo, una visión alternativa de las ideas de los alumnos es coherente con el constructivismo didáctico, para el que estas ideas constituyen un conocimiento alternativo al conocimiento escolar. Una enseñanza basada en la investigación escolar de problemas relevantes constituye una forma idónea de utilizar didácticamente las ideas de los alumnos. Los instrumentos para su detección se acercan a las ideas espontáneas del alumno y se alejan del formato de examen memorístico.

En resumen, podemos contemplar dos planteamientos muy distintos: de la ausencia de valor epistemológico a la consideración de las ideas de los alumnos como un conocimiento alternativo al escolar.

Por otra parte, no son muchas las investigaciones que informen de lo que los profesores piensan y hacen en relación a las ideas de los alumnos. Algunos estudios han detectado progresiones en las concepciones de los profesores sobre lo que son estas ideas, cómo pueden cambiar y cómo se utilizan en el aula (Haefner y Zembal-Saul, 2004), y también hay estudios en los que apenas se detectan progresiones (Park, Hewson y Lemberger, 2010), pero aún hay menos evidencias empíricas de cómo son los instrumentos que los profesores elaboran para detectar las ideas de los alumnos. Este es precisamente el objeto del estudio que presentamos.

\section{Diseño del estudio}

Nos planteamos investigar dos cuestiones: ¿Cómo es el instrumento para detectar las ideas de los alumnos que elaboran diversos equipos de maestros en formación inicial? y ¿cómo progresa en un determinado contexto formativo?

El estudio se realizó a lo largo de un curso basado en la expresión y discusión de las concepciones de los participantes y su contraste con los resultados de la investigación e innovación didácticas. Para ello tomamos en consideración diversas propuestas de formación de profesores de orientación constructivista (Gustafson y Rowell, 1995; Watters y Ginns, 2000; Zembal-Saul, Blumenfeld y Krajcik, 2000), que se basan en actividades en las que los futuros profesores expresan y cuestionan sus concepciones en relación con los problemas prácticos del currículum (Peterson y Treagust, 1998). De forma similar al ciclo de formación propuesto por Zembal-Saul, Blumenfeld y Krajcik (2000), diseñamos una secuencia de actividades formativas con tres momentos:

- El Momento Inicial, para activar, tomar conciencia y elaborar los puntos de vista de los participantes sobre el problema planteado.

- El Momento Intermedio, para provocar contraste y reflexión argumentada entre sus puntos de vista y otras informaciones seleccionadas. 
- Y el Momento Final, para reelaborar los puntos de vista iniciales y explicar las razones de los cambios realizados.

Durante el curso, se abordaron tres problemas prácticos:

- ¿Qué ideas tienen los alumnos de Primaria sobre los contenidos escolares de ciencias? (I)

- ¿Qué contenidos concretos se deben enseñar teniendo en cuenta las ideas de los alumnos? (II).

- ¿Qué plan de actividades puede favorecer que evolucionen las ideas de los alumnos? (III)

El curso de formación comienza con la elaboración de un instrumento para detectar las ideas de los alumnos sobre contenidos escolares relacionados con las ciencias. Los futuros maestros, organizados en equipos de 4 a 6 componentes, elaboraron un instrumento para explorar las ideas de 50 alumnos de Primaria sobre un contenido concreto del currículo de ciencias de su elección. El formador sólo procuró que los tópicos fueran realmente de su interés, que no se repitieran y que, efectivamente, se trataran en Primaria. Cada instrumento fue sometido a revisión mediante el contraste con otros ejemplos y orientaciones prácticas, para después reflexionar sobre los cambios que se deberían hacer en la primera versión del instrumento. Posteriormente, cada equipo administró el instrumento "mejorado" a un grupo de alumnos de Primaria. Los resultados que se presentan en este artículo se corresponden con esta primera parte del problema I.

Una vez obtenidos los instrumentos cumplimentados por los alumnos de Primaria, los equipos seleccionaron diez al azar para realizar un estudio piloto que analizara las ideas de dichos alumnos. Los análisis se sometieron a discusión, contraste con casos de análisis similares, y reflexión, de forma que los equipos realizaron el análisis completo de toda la muestra de alumnos de Primaria y obtuvieron una respuesta al problema I. Posteriormente realizaron una primera propuesta de contenidos (problema II) y de actividades (problema III) que fue sometida a un proceso similar de contraste, reflexión y reelaboración. Una descripción más en detalle del resto del curso puede consultarse en Porlán et al. (2010), así como los resultados del estudio (Porlán et al., 2011).

\section{Participantes}

El curso descrito fue desarrollado por tres formadores de profesores en las Facultades de Educación de dos universidades públicas españolas diferentes, pero con un plan de estudios semejante, sobre aspectos relacionados con la enseñanza y el aprendizaje de las ciencias en Primaria. Participaron un total de 149 estudiantes de Magisterio, mayoritariamente mujeres (90\%), de entre 18 y 25 años. Los resultados que aquí se presentan se refieren a tres equipos (A, B y C), seleccionados al azar, uno por cada uno de los grupos de los formadores. Recordamos que para el desarrollo del curso los equipos seleccionaron libremente un contenido del currículo de ciencias de Primaria. En los equipos estudiados, las temáticas fueron: la reproducción humana (equipo A), los animales (equipo B) y el universo (equipo C).

\section{Obtención y análisis de los datos}

Los datos sobre el instrumento elaborado para detectar las ideas de los alumnos se obtuvieron en los tres momentos mencionados: inicial (primera versión del instrumento), intermedio (al someterlas a contraste y reflexionar sobre ello) y final (versión final del instrumento).

Los equipos, de manera colectiva y previo trabajo individual, elaboraron dos tipos de documentos escritos: de producción libre (versión inicial y final del instrumento) y de reflexión sobre el instrumento utilizado para poner de manifiesto lo que quieren cambiar de la primera versión.

Para caracterizar el instrumento que elaboran los futuros maestros para detectar las ideas de los alumnos, seleccionamos las siguientes categorías de análisis: 
- Lenguaje utilizado en el instrumento

- Forma de preguntar

- Contenido de la pregunta

- Nivel de organización de la realidad a la que se refiere la pregunta

- Grado de apertura en la formulación de la pregunta

- Recurso comunicativo utilizado

- Demanda intelectual que requiere la respuesta

Dichas categorías se seleccionaron a la luz de un primer análisis del contenido del instrumento inicial, siguiendo las recomendaciones de Bardin (1986) y teniendo presente que se trataba de analizar si el instrumento permitía explorar las ideas espontáneas de los alumnos o por el contrario se trataba de una especie de examen recordatorio de lo que habían tratado en clase. Cada una de las categorías se formuló en tres niveles de complejidad creciente. El primer nivel refleja nuestra previsión sobre las concepciones de los maestros en formación al principio del curso (nivel N1) y está basado en estudios previos que ponen en evidencia el predominio de un modelo transmisivo (Porlán, Rivero y Martín del Pozo, 1998).

$\mathrm{El}$ instrumento para acceder a las ideas de los alumnos se basa en preguntas relacionadas con los datos, las definiciones y el lenguaje de una cultura académica. El segundo nivel es un nivel intermedio, que describe las concepciones que esperamos que los futuros maestros alcancen mayoritariamente al final de su participación en el curso de formación inicial (nivel N2), teniendo muy presente lo que es posible alcanzar y que no siempre coincide con lo deseable, por nuestra experiencia como formadores. El último nivel es el de mayor complejidad (nivel N3) para estas categorías, que se corresponde con la visión del constructivismo didáctico que es, desde nuestro punto de vista, el nivel deseable, aunque no siempre sea posible alcanzarlo en determinados contextos formativos. Estos tres niveles de progresión (N1, N2 y N3) constituyen lo que ZembalSaul, Krajcik y Blumenfeld (2002) denominan levels of representation del conocimiento de los futuros profesores. En la Tabla 1 se concretan los tres niveles para las categorías estudiadas.

Tabla 1

Niveles de complejidad en las concepciones de los futuros maestros sobre el instrumento para detectar las ideas de los alumnos (Tomado de Porlán et al., 2011).

\begin{tabular}{|c|l|l|l|}
\hline \multirow{2}{*}{ CATEGORÍAS } & \multicolumn{3}{|c|}{ Niveles de complejidad } \\
\cline { 2 - 4 } & Nivel inicial (N1) & Nivel posible (N2) & $\begin{array}{c}\text { Nivel de referencia } \\
\text { (N3) }\end{array}$ \\
\hline $\begin{array}{l}\text { Lenguaje } \\
\text { utilizado }\end{array}$ & $\begin{array}{l}\text { No apropiado para la } \\
\text { edad de los alumnos. }\end{array}$ & $\begin{array}{l}\text { Se mezcla un lenguaje } \\
\text { apropiado para la edad } \\
\text { de los alumnos con } \\
\text { Otro que no lo es. }\end{array}$ & $\begin{array}{l}\text { Apropiado para la edad } \\
\text { de los alumnos } \\
\text { (accesible, próximo, } \\
\text { cotidiano, etc.) }\end{array}$ \\
\hline $\begin{array}{l}\text { Forma de } \\
\text { preguntar }\end{array}$ & Directa. & $\begin{array}{l}\text { Se mezclan preguntas } \\
\text { directas e indirectas. }\end{array}$ & Indirecta. \\
\hline
\end{tabular}


Tabla 1. (Cont.)

Niveles de complejidad en las concepciones de los futuros maestros sobre el instrumento para detectar las ideas de los alumnos (Tomado de Porlán et al., 2011).

\begin{tabular}{|c|c|c|c|}
\hline \multirow[b]{2}{*}{ CATEGORÍAS } & \multicolumn{3}{|c|}{ Niveles de complejidad } \\
\hline & Nivel inicial (N1) & Nivel posible (N2) & $\begin{array}{l}\text { Nivel de referencia } \\
\text { (N3) }\end{array}$ \\
\hline $\begin{array}{c}\text { Contenido de la } \\
\text { pregunta }\end{array}$ & $\begin{array}{l}\text { El contenido se } \\
\text { relaciona con datos, } \\
\text { nombres, definiciones } \\
\text { estándar, etc. }\end{array}$ & $\begin{array}{l}\text { El contenido se } \\
\text { relaciona con datos, } \\
\text { nombres, definiciones } \\
\text { estándar, etc. y también } \\
\text { con significados. }\end{array}$ & $\begin{array}{l}\text { El contenido se } \\
\text { relaciona con } \\
\text { significados. }\end{array}$ \\
\hline $\begin{array}{l}\text { Nivel de } \\
\text { organización de la } \\
\text { realidad a la que } \\
\text { se refiere la } \\
\text { pregunta }\end{array}$ & $\begin{array}{l}\text { Macrocosmos, } \\
\text { microcosmos y lo no } \\
\text { comúnmente } \\
\text { perceptible. }\end{array}$ & $\begin{array}{l}\text { Mesocosmos y lo no } \\
\text { directamente } \\
\text { perceptible por el } \\
\text { alumno. }\end{array}$ & $\begin{array}{l}\text { Mesocosmos y de } \\
\text { percepción habitual } \\
\text { o/y de relación del } \\
\text { mesocosmos con los } \\
\text { niveles más próximos } \\
\text { del micro y } \\
\text { macrocosmos. }\end{array}$ \\
\hline $\begin{array}{c}\text { Formulación de la } \\
\text { pregunta }\end{array}$ & $\begin{array}{l}\text { Predominantemente } \\
\text { cerradas. }\end{array}$ & $\begin{array}{l}\text { Preguntas abiertas y } \\
\text { cerradas. }\end{array}$ & $\begin{array}{l}\text { Predominantemente } \\
\text { abiertas. }\end{array}$ \\
\hline $\begin{array}{c}\text { Recurso } \\
\text { comunicativo }\end{array}$ & $\begin{array}{l}\text { Sólo texto y muchas } \\
\text { preguntas. }\end{array}$ & $\begin{array}{l}\text { Sólo texto y un } \\
\text { número razonable de } \\
\text { preguntas o dibujos, } \\
\text { personajes, etc. y } \\
\text { muchas preguntas. }\end{array}$ & $\begin{array}{l}\text { Textos, dibujos, } \\
\text { esquemas, personajes, } \\
\text { etc., con un número } \\
\text { razonable de preguntas. }\end{array}$ \\
\hline $\begin{array}{l}\text { Demanda } \\
\text { intelectual que } \\
\text { requiere la } \\
\text { respuesta }\end{array}$ & $\begin{array}{l}\text { Respuestas poco } \\
\text { elaboradas (recordar, } \\
\text { rellenar, señalar, etc.) }\end{array}$ & $\begin{array}{l}\text { Se mezclan preguntas } \\
\text { que requieren } \\
\text { respuestas elaboradas y } \\
\text { poco elaboradas }\end{array}$ & $\begin{array}{l}\text { Respuestas elaboradas } \\
\text { (relacionar, reflexionar, } \\
\text { etc.) }\end{array}$ \\
\hline
\end{tabular}

Para el análisis de estos documentos se siguió un procedimiento con los siguientes pasos:

a) Identificación de las unidades de información significativas que aparecen en los documentos escritos de cada equipo.

b) Clasificación de las unidades de información según las siete categorías de análisis.

c) Elaboración de proposiciones de síntesis, que integran todas las unidades de información de una misma categoría. Esto se repitió en los tres momentos del ciclo formativo (inicial, intermedio y final).

d) Clasificación de cada proposición en uno de los niveles de progresión previstos como hipótesis inicial (N1, N2 y N3). Cuando las proposiciones no encajaban en ninguno de los niveles previstos, se definieron nuevos niveles. Esta situación no ocurrió en el caso de la detección de las ideas de los alumnos, pero sí al estudiar los contenidos escolares o la metodología de enseñanza. Validación de todo el proceso por un sistema de triangulación en el que cada equipo fue analizado independientemente por un mínimo de dos investigadores. Hubo un índice de concordancia de más del 90\% en todos los casos. Las divergencias fueron analizadas separadamente buscando un consenso. 


\section{Resultados}

A continuación, presentamos los resultados de la progresión de cada equipo al elaborar un instrumento para averiguar las ideas de un grupo de alumnos de Primaria sobre un tema de ciencias de su elección (reproducción humana, animales y universo) (ver Tabla 2). La presentación de los resultados se hará atendiendo a los tres momentos de la secuencia formativa (inicial, intermedio y final), para finalmente analizar la progresión detectada.

Tabla 2

Resultados del análisis de los instrumentos elaborados por los equipos $A, B$ y $C$ a lo largo del proceso formativo en cada categoría.

\begin{tabular}{|c|c|c|c|c|c|c|c|c|c|}
\hline CATEGORÍAS & \multicolumn{3}{|c|}{ Momento inicial } & \multicolumn{3}{|c|}{ Momento intermedio } & \multicolumn{3}{|c|}{ Momento final } \\
\hline Lenguaje utilizado & N1 & $\mathrm{N} 2$ & N3 & N3 & N3 & N3 & N3 & $\mathrm{N} 2$ & N3 \\
\hline Forma de preguntar & N1 & N1 & $\mathrm{N} 2$ & --- & --- & --- & N3 & $\mathrm{N} 2$ & $\mathrm{~N} 2$ \\
\hline $\begin{array}{l}\text { Contenido de la } \\
\text { pregunta }\end{array}$ & N1 & N1 & $\mathrm{N} 2$ & --- & N3 & --- & N3 & N3 & N3 \\
\hline $\begin{array}{l}\text { Nivel de organización } \\
\text { de la realidad a la que se } \\
\text { refiere la pregunta }\end{array}$ & $\mathrm{N} 2$ & N3 & $\mathrm{N} 2$ & --- & --- & N3 & N3 & N3 & $\mathrm{N} 2$ \\
\hline $\begin{array}{l}\text { Formulación de la } \\
\text { pregunta }\end{array}$ & N3 & N3 & N3 & N3 & N3 & N3 & N3 & N3 & N3 \\
\hline Recurso comunicativo & N1 & N1 & N1 & N3 & N3 & N3 & N3 & N3 & N3 \\
\hline $\begin{array}{l}\text { Demanda intelectual } \\
\text { que la respuesta requiere }\end{array}$ & N1 & N1 & $\mathrm{N} 2$ & N3 & --- & N3 & N3 & N3 & N3 \\
\hline EQUIPOS & $\mathrm{A}$ & $\mathrm{B}$ & $\mathrm{C}$ & $\mathrm{A}$ & $\mathrm{B}$ & $\mathrm{C}$ & $\mathrm{A}$ & $\mathrm{B}$ & $\mathrm{C}$ \\
\hline
\end{tabular}

\section{Momento inicial}

Inicialmente esperábamos que los equipos de futuros profesores elaboraran un instrumento para averiguar el recuerdo que tenían los alumnos de Primaria sobre los contenidos seleccionados, pero sin considerar las ideas espontáneas de los alumnos. Es decir, un instrumento con un lenguaje muy académico, preguntando de forma directa y cerrada, demandando datos y definiciones, referentes al macro y microcosmos, y que demandara respuestas poco elaboradas, utilizando sólo texto y muchas preguntas (nivel N1).

Pues bien, como puede apreciarse en la Tabla 2, solo el equipo A, en 5 de las 7 categorías responde a este nivel, mientras que el equipo $\mathrm{B}$ lo hace en 4 de ellas y el equipo $\mathrm{C}$ no responde a este nivel, excepto en una categoría (recurso comunicativo).

Tres categorías son destacables. Por una parte, ninguno de los equipos utiliza recursos comunicativos más allá del texto en el momento inicial (nivel N1). El desconocimiento de la potencialidad de los dibujos para detectar ideas puede explicarlo, ya que, como veremos al final, es una de las progresiones más evidentes. Por otra parte, todos los equipos formulan mayoritariamente preguntas abiertas desde el momento inicial (nivel N3). Y, finalmente, todos los equipos hacen referencia al mesocosmos de los alumnos, ya sea con las características del nivel N2 (equipo A) o del nivel N3 (equipos B y C). 
En resumen, como puede apreciarse en el instrumento diseñado en el momento inicial (ver Tabla 3), solo un equipo (A) se sitúa claramente en el nivel N1 con un instrumento más parecido al típico examen de recuerdo, mientras que el equipo B supera dicho nivel por el lenguaje utilizado, por formular preguntas abiertas referentes al mesocosmos, y el equipo C se sitúa en niveles entre N2 y $\mathrm{N} 3$, propios de lo que esperábamos en el momento final.

Tabla 3

Instrumento para detectar las ideas de los alumnos de Primaria sobre la reproducción, los animales y el sistema SolTierra-Luna, elaborado por los equipos en el momento inicial.

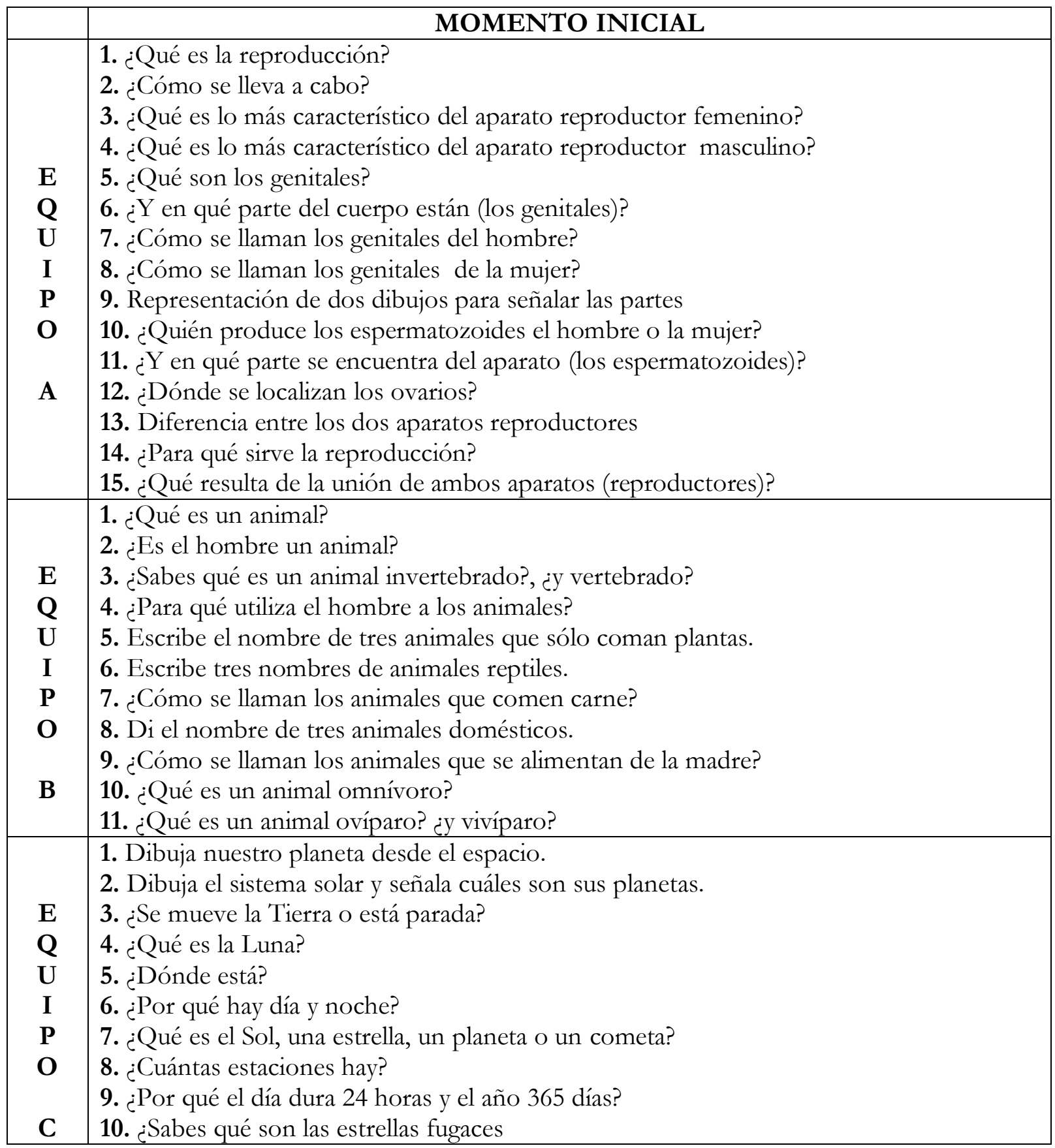


Tabla 3 (Cont.)

Instrumento para detectar las ideas de los alumnos de Primaria sobre la reproducción, los animales y el sistema SolTierra-Luna, elaborado por los equipos en el momento inicial.

11. Si la Tierra es redonda ¿por qué no lo notamos?

12. ¿Por qué la gente no se cae?

13. ¿Qué ocurriría si no hubiera Sol?

14. ¿Por qué los hombres flotan en la Luna?

15. ¿Pueden los hombres respirar en la Luna? ¿por qué?

Nota: en la pregunta 9 se muestra un esquema de las principales estructuras anatómicas internas de los aparatos reproductores masculino y femenino con flechas para relacionarlas con el nombre

\section{Momento intermedio}

Una vez analizados los instrumentos elaborados por todos los equipos en el momento inicial, se contrastan con otros elaborados por maestros innovadores y/o recogidos en la literatura y, los equipos, proceden a reflexionar sobre lo hecho y a plantear posibles cambios. En este momento, esperábamos que los equipos asumieran, al menos parcialmente, las características de un instrumento para detectar las ideas espontáneas de los alumnos y no solo para averiguar lo que recuerdan de lo enseñado. Es decir, un instrumento con un lenguaje menos académico y más cercano a los alumnos, preguntando también de forma indirecta y abierta, demandando significados y no solo datos y definiciones, referentes al mesocosmos y que requieren respuestas más elaboradas utilizando dibujos y no solo texto, y con menos preguntas, pero de más potencialidad para obtener información de lo que piensan los alumnos (nivel N2).

En resumen, todos los equipos se sitúan en el nivel N3, es decir, por encima del que esperábamos en el momento final. No obstante, hay que resaltar que se trata de declaraciones y no de diseños, como sucede en los momentos inicial y final.

\section{Momento final}

Una vez que los equipos tomaron sus decisiones acerca de cómo querían mejorar el instrumento inicial, lo llevaron a la práctica y diseñaron una versión mejorada. Esperábamos que los equipos elaboraran un instrumento característico del nivel N2, que como ya hemos visto asume parcialmente las características de un instrumento para detectar las ideas espontáneas de los alumnos.

Sin embargo, los tres equipos fueron capaces de llevar adelante sus reflexiones y situarse claramente en el nivel N3 (ver Tabla 1): el equipo A, en todas las categorías, y los equipos B y C en todas excepto en la forma de preguntar, en la que siguen manteniendo preguntas directas, en la utilización de algunos términos académicos (equipo B) o en preguntas del nivel no perceptible para los alumnos (equipo C).

En resumen, como puede apreciarse en el instrumento diseñado en el momento final (ver Tabla 4), todos los equipos se sitúan claramente en el nivel N3, con un instrumento idóneo para detectar las ideas espontáneas de los alumnos sobre las temáticas seleccionadas. 
Tabla 4.

Instrumento para detectar las ideas de los alumnos de Primaria sobre la reproducción, los animales y el sistema SolTierra-Luna, elaborado por los equipos en el momento final.

\begin{tabular}{|c|c|}
\hline & MOMENTO FINAL \\
\hline $\begin{array}{l}\mathrm{E} \\
\mathrm{Q} \\
\mathrm{U} \\
\mathrm{I} \\
\mathrm{P} \\
\mathrm{O}\end{array}$ & $\begin{array}{l}\text { 1. En cada una de estas dos figuras (aparecen dos siluetas en blanco): dibuja lo que le falta a cada } \\
\text { una de ellas para ser un hombre y a la otra para ser una mujer. } \\
\text { Escribe el nombre de lo que has dibujado } \\
\text { ¿Para qué sirve cada cosa que has hecho? } \\
\text { 2. Imagina que tus padres se van de viaje. Cuando vuelven tu madre te dice que está embarazada } \\
\text { ¿Qué crees que pasó? } \\
\text { 3. Jaime tiene } 8 \text { años, y no sabe qué parejas pueden tener hijos ¿Puedes ayudarle a resolver su duda? } \\
\text { Hombre - Hombre: si } \square \text { no } \square \text { Porque... } \\
\text { Mujer - Mujer: si } \square \text { no } \square \text { Porque... } \\
\text { Hombre - Mujer: si } \square \text { no } \square \text { Porque... }\end{array}$ \\
\hline & $\begin{array}{l}\text { 1. Clasifica a estos animales y explica por qué lo has hecho así: cabra, león, perro, delfin, mosquito, } \\
\text { paloma, mariposa, tortuga, rana, lagartija, serpiente, lobo zorro y jirafa. } \\
\text { 2. Imagina que cuando seas mayor trabajas en un zoológico y tienes que cuidar a tres animales que } \\
\text { son: una jirafa, un lobo y un oso. Explica qué le darías de comer a cada uno de ellos, y cómo sería el } \\
\text { lugar en el que éstos vivirian. Realiza un dibujo de estos lugares. } \\
\text { 3. Seguro que has visto la película "Liberad a Willy". En esta película un niño es muy amigo de su } \\
\text { ballena llamada Willy, pero cuando se va al mar a nadar con ella, no puede aguantar el mismo } \\
\text { tiempo debajo de agua que la ballena. ¿Por qué le ocurre esto al niño? } \\
\text { 4. Aunque todos los vertebrados pertenecen a un mismo grupo, entre ellos hay grandes diferencias. } \\
\text { Por ejemplo, la ardilla tiene el cuerpo cubierto de pelo y el pájaro carpintero tiene plumas. ¿Por qué } \\
\text { crees que ocurre esto? } \\
\text { 5. Imagina que un día viendo la televisión escuchas que han desaparecido todos los animales que } \\
\text { existen en el mundo, incluyendo los animales que tenemos en casa ¿Qué crees que pasaría? 6. Lee la } \\
\text { adivinanza, di qué animal es este molusco y si es vertebrado o invertebrado. Explica por qué. "Soy } \\
\text { lento y voy tranquilo / porque al andar, mi casa / va conmigo". }\end{array}$ \\
\hline & $\begin{array}{l}\text { 1. Dibuja en los recuadros (aparecen dos recuadros en blanco con cuatro líneas debajo para escribir y } \\
\text { con la palabra DIA y NOCHE encima de cada uno) lo que ves en el cielo durante el día y durante } \\
\text { la noche. Debajo, comenta con tus palabras el dibujo que has realizado. } \\
\text { 2. (Aparece el extraterrestre en pequeño y seis líneas para escribir) Hace mucho tiempo, cuando se } \\
\text { formó, el universo no era igual que ahora. En mi planeta hemos inventado una máquina del tiempo y } \\
\text { queremos retroceder al momento en que se formó. ¿Qué crees que nos encontraremos? } \\
\text { 3. (Aparece un recuadro grande sin lineas y debajo cuatro afirmaciones) Me gustaría saber cómo } \\
\text { piensas que es una galaxia. Dibújala y elige una de estas frases para decirme qué es una galaxia:- } \\
\text { Una galaxia es un conjunto de meteoritos y estrellas.-Una galaxia es un conjunto de estrellas, } \\
\text { satélites, planetas y meteoritos.-Una galaxia es un conjunto de satélites y planetas.-una galaxia es un } \\
\text { conjunto de meteoritos, planetas y satélites. } \\
\text { 4. (Aparecen sin lineas numeradas del } 1 \text { al } 5 \text { ) Escríbeme } 5 \text { cosas que pasarian en la tierra si alguien } \\
\text { le diera a un botón y se apagara el solpara siempre. } \\
\text { 5. (Aparece el extraterrestre en pequeño y } 6 \text { lineas con la letra V y F delante) Respóndeme verdadero } \\
\text { o falso:-El sol gira alrededor de la tierra.-La tierra gira alrededor del sol.-La tierra gira alrededor de } \\
\text { la luna.-El sol es más grande que la tierra.-El sol se apaga cuando no lo vemos.-La luna siempre la } \\
\text { vemos redonda. ¿Por qué? }\end{array}$ \\
\hline
\end{tabular}


Tabla 4 (Cont.)

Instrumento para detectar las ideas de los alumnos de Primaria sobre la reproducción, los animales y el sistema SolTierra-Luna, elaborado por los equipos en el momento final.

6. (Aparece el extraterrestre en pequeño, un recuadro grande y debajo cuatro lineas para escribir) Dibuja el sistema
solar y di qué cosas se mueven y cuáles están quietas.
7. (Aparece un recuadro grande) Imagina que eres un astronauta y que estás en el espacio. Dibuja cómo crees qué
verías la tierra desde alliy dónde está la gente.
8. (Aparece el extraterrestre en pequeño y cinco líneas para escribir) En mi planeta siempre es invierno en todas partes,
y hace mucho frío. Pero en la tierra, cuando en algunos países es invierno y hace frío, en otros países es verano y hace
calor. ¿Por qué piensas que pasa esto?
9. (Aparecen seis viñetas y cuatro líneas debajo de cada una de ellas para escribir) Imaginate que vienes conmigo en mi
nave y damos una vuelta por el espacio. Haz un cómic de cómo veríamos desde allí cómo se hace de día y de noche en la
tierra. Utiliza los recuadros que necesites y comenta lo que dibujas.

\section{Discusión}

Por los resultados que acabamos de presentar, parece confirmarse que los futuros maestros, en el contexto del curso de formación, asumen los planteamientos esenciales que caracterizan un instrumento de detección de las ideas espontáneas de los alumnos (nivel N3). Esto puede estar relacionado, a nuestro entender, con la superación de un obstáculo de carácter psicológico-didáctico (Porlán et al., 2011; Rivero et al., 2011; Martín del Pozo, Rivero y Azcárate, 2014), en el que se toma en consideración el punto de vista del alumno o, dicho en otros términos, la cultura relacionada con la edad frente a la cultura académica. En esta perspectiva hay una preocupación por adaptar la pregunta con el fin de estimular a los alumnos para responder con lo que piensan y no con lo que se supone que tienen que saber de cursos anteriores. Del mismo modo, las preguntas ya no son vistas como herramientas para comprobar si ellos tienen el conocimiento que se supone que han debido de aprender, sino para averiguar lo que realmente piensan.

Con estrategias muy similares a la nuestra, Hollon, Roth y Anderson (1991) encontraron que la mayoría de los profesores diseñan instrumentos de bajo nivel para explorar las ideas de los alumnos. Incluso Morrison y Lederman (2003) hallaron que ninguno de los profesores participantes en su estudio mencionó el uso de instrumentos para detectar las ideas de los alumnos en el aula, a pesar de que declararon que es importante diagnosticarlas. También Tabachnick y Zeichner (1999) encontraron que aunque la mayoría de los futuros profesores adquirieron experiencia en la detección de las ideas de los alumnos, sólo unos pocos fueron capaces de planificar su enseñanza de acuerdo con esas ideas. Por ello, estamos de acuerdo con Gustafson y Rowell (1995) cuando afirman que probablemente un cambio más consistente (no solo ser capaces de elaborar un instrumento) sólo es posible en un contexto del ejercicio de la práctica profesional. Para nosotros ese cambio sería pensar y utilizar las ideas de los alumnos dentro del marco del constructivismo didáctico (nivel N3).

\section{Conclusiones}

Lejos de nuestras previsiones iniciales, podemos concluir que los futuros maestros analizados progresan en el diseño del instrumento, desde algo parecido a un examen de recuerdo (equipo A en el nivel N1) o desde instrumentos intermedios (equipos B y C en niveles N1, N2 y N3, según las categorías) a instrumentos diseñados para poner de manifiesto las ideas espontáneas de los alumnos (equipos A, B y C en el nivel N3). 
Los resultados del estudio nos han permitido proponer una hipótesis más fundamentada en datos empíricos, integrando los niveles del mismo grado de complejidad de las siete categorías estudiadas. Podemos concluir proponiendo un Itinerario de Progresión (Solís, Porlán y Rivero, 2012) en relación al instrumento para detectar las ideas de los alumnos, diferenciando dos niveles generales:

a) En el nivel de menor complejidad, que denominamos de Cultura Académica, el instrumento para detectar las ideas de los alumnos se basa en preguntas directas y cerradas, con un lenguaje muy académico, sobre datos y definiciones, referentes al macro y microcosmos y que requieren respuestas poco elaboradas, utilizando texto y muchas preguntas. Lo más parecido a un examen memorístico. Se trata de averiguar los conocimientos escolares que el alumno es capaz de recordar. El obstáculo que parece reforzar esta posición es la creencia de que las ideas de los alumnos son aquello que recuerdan de la enseñanza recibida. La actividad formativa que ha podido tener más influencia para superar este nivel es el hecho de poder comparar sus propuestas con otras propuestas alternativas sobre la misma temática, donde se apreciaba un lenguaje no académico, situaciones cercanas sobre las que preguntar a los alumnos, dibujos, etc., y reflexionar sobre lo que han hecho inicialmente.

b) El nivel de mayor complejidad, que denominamos de Cultura de Edad, es el nivel de referencia. El instrumento para detectar las ideas de los alumnos se basa en pocas preguntas que presentan situaciones cercanas a los alumnos y son abiertas, con un lenguaje adecuado y cercano a los alumnos, sobre significados referentes al mesocosmos y que requieren respuestas elaboradas, utilizando la potencialidad de los dibujos y no solo texto. Se trata pues de averiguar las ideas espontáneas de los alumnos.

No obstante, por los resultados obtenidos, hemos de señalar que entre ambos niveles hay un nivel intermedio, en el que se supera la cultura académica y se considera la cultura de edad, pero parcialmente, dependiendo del contenido escolar seleccionado y de la categoría que consideremos. Por ejemplo, tiene una especial dificultad para los futuros profesores elaborar preguntas indirectas (los equipos B y C no han pasado del nivel N2), es decir, plantear situaciones a través de las cuáles podamos obtener datos de las ideas espontáneas de los alumnos de Primaria. Sin embargo, el equipo A ha logrado elaborar una pregunta en el instrumento final (la $\left.n^{\circ} 1\right)$ que incluye la información de nueve preguntas del instrumento inicial.

Este Itinerario de Progresión puede representarse en forma de una escalera en la que podemos visualizar los niveles, el obstáculo que dificulta la progresión de uno a otro, y las actividades formativas que pretenden facilitar la progresión (ver Figura 1). 


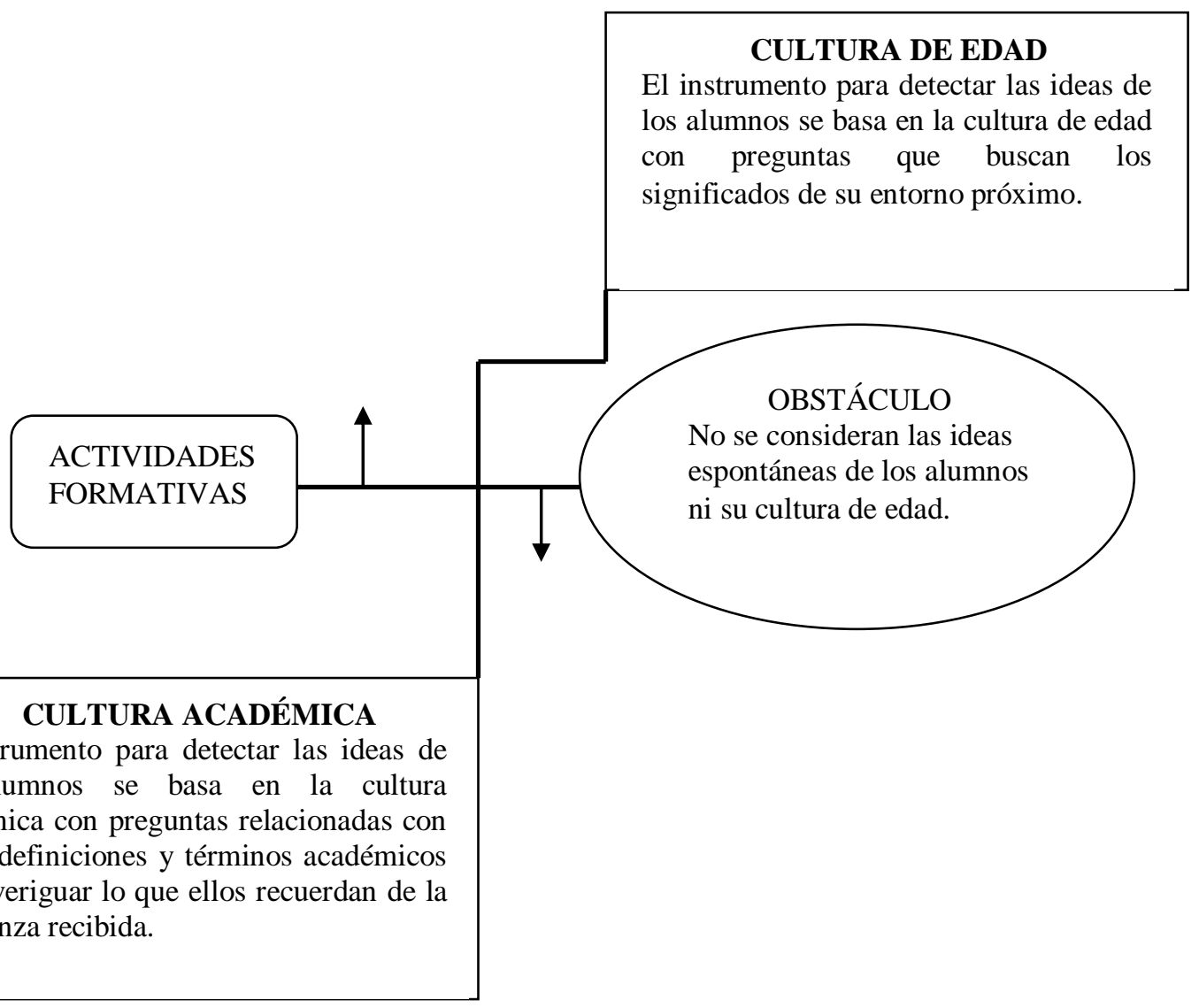

Figura 1. Itinerario General de Progresión sobre el instrumento para detectar las ideas de los alumnos (elaborado a partir de Porlán et al., 2011)

\section{Implicaciones en la formación inicial del profesorado}

La principal implicación del presente estudio en la formación inicial de maestros de Primaria es que el hecho de plantear una situación de la práctica profesional "real" como es elaborar un instrumento para averiguar las ideas de alumnos "reales" sobre un contenido "real" del currículo, es lo que puede haber contribuido a alcanzar la progresión detectada. Así pues, organizar el currículo de la formación de maestros en torno a problemas prácticos profesionales da sentido a la formación inicial. El hecho de trabajar sobre situaciones vinculadas con la práctica futura y, reflexionar sobre cómo abordarlas, permite a los estudiantes de Magisterio ponerse en la situación del docente y tomar decisiones, reflexionado sobre cuáles son las más adecuadas y por qué, contrastando sus propias visiones con prácticas alternativas y no sólo con informaciones teóricas (Duit y Treagust, 2003). No podemos olvidar que los únicos referentes prácticos directos que poseen los futuros maestros son aquellos que han vivido como alumnos, es la única práctica educativa que conocen y en ella se basan, aún sin ser conscientes, para diseñar y desarrollar su enseñanza. Por ello necesitan experimentar sus nuevas ideas y reflexionar sobre dicha experimentación y así poder ir consolidando las progresiones (Watts y Jofili, 1998; Tillema 2000; Zembal-Saul, Krajcik y Blumenfeld, 2002).

Por último, señalar que estamos profundizando en este estudio con más equipos de futuros 
maestros para ver la consistencia de la progresión detectada, la utilidad de las categorías seleccionadas para caracterizar el instrumento y la utilidad del Itinerario de Progresión propuesto. Además, hemos aumentado la interacción con la práctica real, incluyendo el visionado y análisis de videos de maestros de Primaria trabajando en clase con las ideas de sus alumnos, para tratar de consolidar y mejorar las progresiones detectadas en este estudio. ${ }^{1}$

\section{Referencias}

Bardin, L. (1986). Análisis de contenido. Madrid: Akal.

Duit, R. \& Treagust, D. (2003) Conceptual change: A powerful framework for improving science teaching and learning. International Journal of Science Education, 25(6), 671-688.

Giordan, A. (1989) Representaciones sobre la utilización didáctica de las representaciones. Enseñanza de las Ciencias, 7(1), 53-62.

Gustafson, B.F. \& Rowell, P.M. (1995). Elementary preservice teachers: constructing conceptions about learning science, teaching science and the nature of science. International Journal of Science Education, 17, 589-605.

Hollon, R.E, Roth, K. \& Anderson, C.W. (1991). Science teachers' conceptions of teaching and learning. Advances on Research on Teaching, 2, 145-185.

Jones, M., Carter, G. \& RUA, M. (1999) Children's concepts: tools for transforming science teachers' knowledge. Science Education, 83(5), 545-557.

Joram, E.\&Gabriele, A.J. (1998). Preservice teacher's prior beliefs: transforming obstacles into opportunities. Teaching and Teacher Education, 14(2), 175-191.

Martín Del Pozo, R., Porlán, R. \& Rivero, A. (2011). The progression of prospective teachers' conceptions of school science content. Journal of Science Teacher Education. Published online: 27 Abril 2011.

Martín Del Pozo, R., Rivero, A. \& Azcárate, P. (2014). Las concepciones de los futuros maestros sobre la naturaleza, cambio\&utilización didáctica de las ideas de los alumnos. Revista Eureka sobre Enseñanza y Divulgación de las Ciencias, 11(3), 348-363.

Morrison, J. A. \& Lederman, N. G. (2003). Science teachers' diagnosis and understanding of students' preconceptions. Science Education, 87(6), 849-867.

Park, H., Hewson, P. W. \& Lemberger, J. (2010). The interactions of conceptions of teaching science and environmental factors to produce praxis in three novice teachers of science. Research in Science Education, 40, 717-741.

Peterson, R. \& Treagust, D. (1998). Learning to teach Primary Science through problem-based learning. Science Education, 82, 215-237.

Porlán, R., Rivero, A. \& Martín Del Pozo, R. (1998). Conocimiento profesional\&epistemología de los profesores II: Estudios empíricos y conclusiones. Enseñanza de las Ciencias, 16(2), 271-288.

Porlán, R., Martín Del Pozo, R., Rivero, A., Harres, J., Azcárate, P. \& Pizzato, M. (2010). El cambio del profesorado de ciencias I: Marco teórico y formativo. Enseñanza de las Ciencias, 28(1), 31 46.

\footnotetext{
${ }^{1} \mathrm{El}$ estudio presentado forma parte, como estudio piloto, del proyecto $\mathrm{I}+\mathrm{D}+\mathrm{i}$ : La progresión del conocimiento didáctico de los futuros profesores en un curso basado en la investigación y en la interacción con una enseñanza innovadora de las ciencias (EDU201123551), financiado por el Ministerio de Ciencia e Innovación (hoy de Economía y Competitividad).
} 
Porlán, R., Martín Del Pozo, R., Rivero, A., Harres, J., Azcárate, P. \& Pizzato, M. (2011). El cambio del profesorado de ciencias II: Resultados\&conclusiones sobre la progresión de las concepciones didácticas. Enseñanza de las Ciencias, 29(3), 413-426.

Rivero, A., Azcárate, P., Porlán, R., Martín Del Pozo, R.\&Harres, J. (2010). The Progression of Prospective Primary Teachers' Conceptions of the Methodology of Teaching. Research in Science Education. Published online: 29 Septiembre 2010.

Solís, E., Porlán, R.\&Rivero, A. (2012) ¿Cómo representar el Conocimiento Curricular de los profesores de Ciencias y su evolución? Enseñanza de las Ciencias, 30(3), 9-30

Solís, E., Porlán, R., Rivero, A. \& Martín Del Pozo, R. (2012). Las concepciones de los profesores de ciencias de Secundaria en formación inicial sobre metodología de enseñanza. Revista Española de Pedagogía, 253, 495-514.

Tabachnick, B.R. \& Zeichner, K.M. (1999). Idea and action: Action research and the development of conceptual change teaching of science. Science Education, 83, 309-322.

Tillema, H.H. (2000). Belief change towards self-directed learning in student teachers: immersion in practice or reflection on action. Teaching and Teacher Education, 16(5/6), 575-591.

Treagust, D. \& Duit, R. (2008) Conceptual change: a discussion of theoretical, methodological and practical challenges for science education. Cultural Studies in Science Education, 3, 297-328.

Watters, J. J. \& Ginns, I. (2000). Developing motivation to teach elementary science: effect of collaborative and authentic learning practices in preservice education. Journal of Science Teacher Education, 11(4), 301-321.

Watts, M. \& Jofili, Z. (1998) Towards critical constructivist teaching. International Journal of Science Education, 20(2), 173-185.

Zembal-Saul, C., Blumenfeld, P. \& Krajcik, J. (2002). Influence of guided cycles of planning, teaching, and reflection on prospective elementary teachers' science content representations. Journal of Research in Science Teaching, 37, 318-339.

\section{Sobre los Autores}

Autor: Emilio Solís Ramírez

Institución: Facultad de Ciencias de la Educación. Universidad de Sevilla

E-mail: esolis@us.es

Información biográfica: Licenciado en Ciencias Químicas por la Universidad de Granada. Doctor en Ciencias de la Educación por la Universidad de Sevilla. Profesor asociado del Departamento de Didáctica de las Ciencias Experimentales de la Universidad de Sevilla y profesor en el Instituto Provincial de Educación Permanente de Sevilla. Miembro del grupo de investigación y del colectivo iberoamericano de profesoras/es Red IRES (Investigación y Renovación Escolar). Línea de investigación: formación del profesorado de ciencias.

ORCID: 0000-0002-5595-6283

Autor: Rafael Porlán Ariza

Institución: Facultad de Ciencias de la Educación. Universidad de Sevilla

E-mail: rporlan@us.es

Información biográfica: Licenciado en Ciencias Biológicas por la Universidad de Sevilla. Doctor en Ciencias de la Educación por la Universidad de Sevilla. Catedrático del Departamento de Didáctica de las Ciencias Experimentales de la Universidad de Sevilla. Co ordinador del Grupo de Investigación HUM 319 - DIE (Didáctica e Investigación Escolar)- de la Junta de 
Andalucía, investigación y del colectivo iberoamericano de profesoras/es Red IRES (Investigación y Renovación Escolar). Línea de investigación: formación del profesorado de ciencias.

ORCID: 0000-0003-2068-7092

Autor: Rosa Martín del Pozo

Institución: Facultad de Educación. Universidad Complutense de Madrid.

E-mail: rmartin@edu.ucm.es

Información biográfica: Licenciada en Ciencias Químicas y Ciencias de la Educación. Doctora en Ciencias de la Educación. Profesora Titular del Departamento de Didáctica de las Ciencias Experimentales de la Universidad Complutense de Madrid. Miembro del Grupo de Didáctica e Investigación Escolar (DIE). Codirectora del Grupo de Investigación de la UCM sobre Competencias docentes. Línea de investigación: conocimiento profesional de los profesores de ciencias.

ORCID: no disponible

Autor: Joao Batista Harres

Institución: Facultad de Física. Universidad Católica do Rio Grande do Sul (Brasil)

E-mail: joao.harres@pucrs.br

Información biográfica: Licenciada en Física. Doctor en Educación. Profesor Adjunto de Facultad de Física de la Universidad Católica do Rio Grande do Sul (Brasil). Miembro del Grupo de Investigación en Enseñanza de la Física. Línea de investigación: Enseñanza, aprendizaje y formación de profesores.

ORCID: http://lattes.cnpq.br/7625130635800187

\section{Investigación en la Escuela}

Revista académica evaluada por pares y de acceso abierto

Número 88

1 de octubre de 2016

ISSN 2443-9991

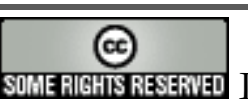

ST se de crédito y atribución al autor/es y a Investigación en la Escuela, se distribuya con propósitos no-comerciales, no se altere o transforme el trabajo original. Más detalles de la licencia de Creative Commons se encuentran en http://creativecommons.org/licenses/by-nc-sa/3.0 Cualquier otro uso debe ser aprobado en conjunto por el autor/es, o Investigación en la Escuela.

Contribuya con comentarios y sugerencias en la web de la revista. Por errores y sugerencias contacte a investigacionescuela@,ddcc.uhu.es 


\title{
Investigación en la escuela
}

Consejo de dirección: Ana Rivero García (Universidad de Sevilla), Nicolás de Alba Fernández (Universidad de Sevilla), Pedro Cañal de León (Universidad de Sevilla), Francisco F. García Pérez (Universidad de Sevilla), Gabriel Travé González, (Universidad de Huelva), Francisco F. Pozuelos Estrada (Universidad de Huelva)

\author{
Dirección: Ana Rivero García y Nicolás de Alba Fernández \\ Técnico de edición: Francisco Javier López Sánchez
}

\section{Consejo editorial}

José Félix Angulo Rasco. Universidad de Cádiz Rosa Ma Ávila Ruiz. Universidad de Sevilla Pilar Azcárate Goded. Universidad de Cádiz Juan Bautista Martínez Rodríguez. Universidad de Granada

Nieves Blanco García. Universidad de Málaga Fernando Barragán Medero. Universidad de La Laguna José Carrillo Yáñez. Universidad de Huelva José Contreras Domingo. Universidad de Barcelona. Luis C. Contreras González. Universidad de Huelva Ana $\mathbf{M}^{\mathbf{a}}$ Criado García-Legaz. Universidad de Sevilla Rosario Cubero Pérez. Universidad de Sevilla José $\mathbf{M}^{\mathbf{a}}$ Cuenca López. Universidad de Huelva Jesús Estepa Giménez. Universidad de Huelva Rafael Feito Alonso. Universidad Complutense (Madrid)

Francisco José García Gallardo. Universidad de Huelva

Soledad García Gómez. Universidad de Sevilla J. Eduardo García Díaz. Universidad de Sevilla
Fernando Hernández Hernández. Universidad de Barcelona

Salvador Llinares Ciscar. Universidad de Alicante Alfonso Luque Lozano. Universidad de Sevilla Rosa Martín del Pozo. Universidad Complutense (Madrid)

José Martín Toscano. IES Fernando Herrera (Sevilla) Jaume Martínez Bonafé. Universidad de Valencia F. Javier Merchán Iglesias. Universidad de Sevilla Emilia Moreno Sánchez. Universidad de Huelva. Rosario Ortega Ruiz. Universidad de Córdoba Antonio de Pro Bueno. Universidad de Murcia Fco. de Paula Rodríguez Miranda. Universidad de Huelva

Pedro Sáenz-López Buñuel. Universidad de Huelva Antoni Santisteban Fernández. Universidad Autónoma (Barcelona)

Emilio Solís Ramírez. Catedrático de IES.

$\mathbf{M}^{\mathbf{a}}$ Victoria Sánchez García. Universidad de Sevilla. Magdalena Suárez Ortega. Universidad de Sevilla

\section{Consejo asesor}

Manuel Area Moreira. Universidad de La Laguna

Jaume Carbonell. Director Cuadernos de Pedagogía. Barcelona

César Coll. Universidad de Barcelona

Christopher Day. Universidad de Nothingham. U.K.

Juan Delval. Universidad Nacional de Educación a Distancia

John Elliott. Universidad de East Anglia. Norwich. U.K.

José Gimeno Sacritán. Universidad de Valencia

André Giordan. Universidad de Paris VII y Ginebra

Francisco Imbernón. Universidad de Barcelona

Ángel Pérez Gómez. Universidad de Málaga

Rafael Porlán Ariza. Universidad de Sevilla

Francesco Tonucci. Instituto de Pedagogía del C.N.R. Roma

Jurjo Torres Santomé. Universidad de A Coruña 